TRANS · núm. $24 \cdot 2020$

MISCELÁNEA · 319-333
El presente estudio describe una experiencia didáctica colaborativa e innovadora en el campo de la traducción literaria. La experiencia tuvo lugar en la asignatura optativa de Traducción Literaria Avanzada del último curso del Grado en Traducción e Interpretación de la Universidad de Alicante. Se fijó como objetivo literario principal llevar a cabo una traducción doble, en prosa y poética, que pudiera aportar un valor añadido a las ya existentes, ninguna de las cuales presenta esta combinación junto con un análisis retórico individual de cada soneto, lo que permite una lectura múltiple y complementaria de los sonetos. El objetivo didáctico principal consistía en situar al alumnado ante un encargo real para conseguir su empoderamiento (Kiraly, 2001, 2005, 2014). Siempre con la supervisión y retroalimentación del profesor, la metodología se basó en el trabajo horizontal en grupo para fomentar la colaboración y el control de calidad mediante revisión por pares (Pietrzak, 2014) junto con el reparto de roles de editor, traductor, documentalista y corrector dentro de cada grupo. Al mismo tiempo, se acordó la posible publicación del resultado como libro por parte de un editor profesional. Aparte del proceso dialéctico de retroalimentación continua horizontal y vertical antes descrito, se hizo una encuesta anónima de satisfacción y posibles enfoques didácticos alternativos al alumnado en la que se trataba de recoger su percepción de todo el proceso. El proyecto contó además con la evaluación final de los resultados por parte de un agente externo experto, el responsable de la editorial destinataria, para el que la publicación supone una apuesta económica y de prestigio.

PALABRAS CLAVE: enseñanza-aprendizaje colaborativos, traducción, poesía, William Shakespeare.

\title{
Un proyecto colaborativo didáctico y profesionalizante en torno a la traducción de los sonetos de William Shakespeare
}

\author{
JaVIer Franco Aixelé \\ PaOla Masseau \\ IRENE FLORES FueNTES \\ Marina Pérez Bernal
}

Universidad de Alicante

\section{A Collaborative, Didactic and Profession-Oriented Project Based on the Translation of William Shakespeare's Sonnets}

This essay addresses an innovative and collaborative didactic experience in the field of literary translation. The project took place within Advanced Literary Translation, an optional subject in the last year of the Degree in Translation and Interpreting of the University of Alicante. The main literary aim was to provide a multiple and comprehensive approach to Shakespeare's Sonnets from which the readers could attain a detailed view of the poems from various and complementary angles. To this end, we performed a double translation, in prose and verse, completed by a rhetorical analysis and a synopsis of each sonnet. All this was done in order to contribute some added value to already existing translations, none of which provides this combination. The main didactic aim was to place the students in a real professional situation, with a specific commission, in order to empower them (Kiraly, 2001, 2005, 2014). Under the supervision of and with feedback from the teacher, the methodology was based on horizontal group work with the idea that this would enhance collaboration and quality control through peer revision (Pietrzak, 2014). To this, a distribution of specific roles for each translation was added, with students alternatively acting as publisher, translator, documentalist and proof editor in each group. The publication of the results in a real book by a professional publisher was the final result sought. Apart from the dialectic process of horizontal and vertical feedbacks, an anonymous questionnaire was administered to elicit the students' degree of satisfaction and alternative didactic strategies. The project was further sanctioned by an external expert agent, the publisher, for whom the actual launching of the book involved putting his economic resources and prestige at stake.

KEY WORDS: collaborative teaching-learning, translation, poetry, William Shakespeare. 


\section{1. INTRODUCCIÓN}

En el ámbito de las humanidades, el Grado en Traducción e Interpretación se caracteriza por una orientación especialmente profesionalizante, lo que implica un peso específico notable de las prácticas, que típicamente constituyen tres cuartas partes de los créditos de las asignaturas específicas de traducción. Tradicionalmente, esta labor se ha abordado partiendo de la premisa de que el profesor es el experto y su tarea consiste en buscar textos que concentren problemas de traducción interesantes, encargarlos al alumnado y después corregirlos en clase, señalando los errores cometidos y proporcionando «la» traducción correcta. Este procedimiento puede resultar en buena medida efectivo, pero también presenta tres carencias de base que en este proyecto se intentan suplir mediante un enfoque colaborativo que incorpore la perspectiva didáctica de colaboración horizontal y una enseñanza basada en la réplica de situaciones sociolaborales reales e integración progresiva en un contexto comunitario real o situated learning (Lave y Wenger 1991). Se trata de planteamientos didácticos de enfoque constructivista que hicieron su entrada en los estudios de traducción hace ya más de veinte años (Kiraly 1995), pero que todavía no se han consolidado en nuestra docencia universitaria.

En primer lugar, la figura del profesor omnisciente y dueño de la solución única y exacta a los problemas choca frontalmente con el carácter inherentemente variable y dinámico de la traducción. Basta con repasar las retraducciones de cualquier clásico para comprobar de manera palpable que todo texto se puede traducir de maneras múltiples, todas ellas en principio legítimas, dependiendo de factores como la asimetría entre las convenciones genéricas de partida y acogida, el lector tipo al que va destinada la traducción, la función que ha de cumplir el texto traducido o el concepto mismo de traducción, la sensibilidad y las opciones estéticas del propio traductor. Pretender por ello que la versión del profesor sea la única aceptable resulta contrario a la realidad profesional y contraproducente para la formación de un traductor, que perdería flexibilidad, creatividad y una perspectiva holística de la traducción profesional, tal como es.

En segundo lugar, se halla la cuestión del enfoque evaluador únicamente vertical como barrera para el desarrollo de la autonomía o empoderamiento. Con la asignatura de Traducción Literaria Avanzada nos encontramos en la fase final del proceso educativo universitario. $\mathrm{Al}$ año siguiente de concluir la asignatura en la que se enmarca este proyecto se supone que el alumnado estará capacitado para ejercer profesionalmente su labor. La traducción constituye una actividad compleja de interpretación de significados y toma de decisiones constantes en la que el profesional debe proporcionar un resultado final de calidad y listo para utilizar directamente. Para abordarla, precisa de competencias básicas como la de resolver problemas de forma autónoma y, sobre todo, una capacidad de evaluación experta para cuya consecución actuar en clase como receptor pasivo de la valoración del docente presenta claras limitaciones. Resulta de todo punto necesario que el alumnado sea capaz no solo de traducir, sino de juzgar las traducciones de manera autónoma y con criterios objetivos, tanto para decidir entre diversas soluciones como para defender su versión ante el iniciador con argumentos intersubjetivos convincentes. Se trata de una parte de su trabajo que tendrá que aplicar constantemente ante clientes y correctores. Para poder hacerlo bien, resulta más que conveniente que gane la autoestima y confianza imprescindibles (Johnson y Johnson, 1990) a través de un enfoque colaborativo basado en la práctica revisora 
en horizontal, debatiendo con sus compañeros sobre las virtudes y carencias del trabajo realizado a partir de encargos en los que tenga una responsabilidad individual clara (Durán, 2014). El debate con sus pares, libre de la presión de cualquier evaluación académica y del respeto a la autoridad simbolizada por el docente, constituye en este sentido una escuela inestimable a la hora de pensar y defender las motivaciones y resultados de la propia traducción.

En tercer lugar, está el problema del conocimiento de los roles y procedimientos del mercado profesional. Al mismo tiempo que la traducción literaria se basa en una labor eminentemente individual, en su dimensión profesional se halla sujeta a una intensa interacción con los demás agentes responsables de la publicación, especialmente el editor y el corrector de estilo. Una filosofía didáctica que no trascienda la burbuja académica mediante la enseñanza situada (González Davies y Enríquez Raído, 2016) carecerá por tanto de uno de los pilares básicos de la realidad en la que ha de saber desenvolverse el traductor. El enfoque horizontal y colaborativo mediante participación periférica legítima y la asunción de roles acordes con la realidad en una comunidad de prácticas constituye una buena manera de abordar este problema. En palabras de Marco Borillo (2016, 29):

The concepts of situated learning and legitimate peripheral participation (LPP) provide a theoretical framework for learning that is studentcentred, based on collaboration and placed on the margins of a given community of practice, with a view to bringing the learner closer to that community. In translation pedagogy, these features converge most notably with the tenets of social constructivism, which insists on the importance of the publishable translation project in translator training methodology.
Con estas desventajas o limitaciones didácticas del método exclusivamente vertical en mente, el principal objetivo que se ha querido alcanzar con este proyecto consiste en demostrar que el diseño de una experiencia didáctica basada en el constructivismo social, colaborativa, situada y altamente ambiciosa en cuanto a la calidad y repercusión de los resultados finales en el mundo editorial profesional podría concitar un intenso interés y empoderamiento del alumnado, con repercusiones especialmente positivas en tres ámbitos: su autoestima y confianza, su capacidad para discriminar de forma justificada entre diversas traducciones posibles y su capacidad para una autocrítica proactiva, capaz de ofrecer como resultado propuestas de traducción cada vez más conscientes, creativas y sofisticadas.

\section{MÉTODO}

\subsection{Descripción del contexto y de los participantes}

Este proyecto didáctico se llevó a cabo en el seno de la asignatura Traducción Literaria Avanzada Inglés-Español, optativa de seis créditos del último curso del Grado de Traducción e Interpretación de la Universidad de Alicante con 22 alumnas (21 mujeres y un varón). El hecho de que sea una asignatura optativa aporta una disposición inicial especialmente proactiva, mientras que su situación al final de la etapa formativa garantiza tanto una notable conciencia de la necesidad de conocer el mundo profesional como un número importante de horas previas de prácticas de traducción a lo largo de tres cursos de carrera, entre las que destacan tanto las prácticas documentales en una asignatura específica como las de la asignatura de traducción literaria, obligatoria del curso anterior, todo lo cual aporta una base sólida sobre la que construir su prolongación avanzada. Esta doble circuns- 
322 tancia hace que un proyecto profesionalizante de empoderamiento en el que el alumnado se responsabilice de manera central de su propia formación con un fuerte componente horizontal resulte viable. Y, efectivamente, cuando el profesor planteó la iniciativa, la respuesta fue de alto compromiso. En este sentido, debe hacerse constar que en todo momento se ofreció la opción de realizar un trabajo final alternativo de análisis de traducción, menos creativo y exigente, además de muy similar a otros ya hechos en asignaturas anteriores de la carrera. En un primer momento, además, se abordó este proyecto sin garantías de publicación editorial impresa, planteando la posibilidad de que finalmente el resultado se publicase únicamente en la web de la universidad dependiendo de la calidad final que se alcanzara. Esto no supuso ninguna rebaja del entusiasmo inicial y la totalidad del alumnado eligió este proyecto.

\subsection{Instrumentos y procedimiento}

El diseño del proyecto se basó en habilitar al alumnado para poder realizar un intenso proceso de aprendizaje autónomo con un fuerte componente de interacción horizontal mediante trabajo en grupo. A ello se sumaban las sesiones de orientación previa por parte del profesor y su supervisión, con puesta a disposición permanente para guiar y resolver dudas, además de proporcionar retroalimentación en varios momentos claves del proceso para garantizar su viabilidad. Los principales instrumentos y procedimientos utilizados en esta experiencia educativa fueron los siguientes:

- Diseño del proyecto por parte del profesor. Se trataba de buscar para la traducción un autor interesante de dominio público que nos permitiera sortear el problema de los derechos y cuya obra fuese compartimentable en textos independientes relativamente breves. Esto facilitó su distribución entre distintos grupos que trabajarían en gran medida de forma independiente, aunque siempre a partir de un marco común en cuanto a los principales parámetros del proyecto, muy especialmente el tipo de traducción que se iba a llevar a cabo. Por estas razones se eligieron los sonetos de Shakespeare, poemas que se pueden traducir independientemente para una obra colectiva siempre que se establezcan claramente el marco literario interpretativo que unifica el subciclo elegido y unas condiciones de traducción claras y comunes, que además se pudieron debatir y practicar previamente en clase con textos comparables procedentes del mismo volumen original con los 154 sonetos de Shakespeare, como veremos a continuación.

Una consideración importante que se debía tener en cuenta es que los sonetos de Shakespeare se han traducido al español numerosas veces en el pasado, desde finales del siglo xix hasta la actualidad, con versiones parciales o completas, en prosa o en distintos formatos poéticos (fundamentalmente, endecasílabos y alejandrinos, ocasionalmente blancos). Quizá las más emblemáticas (no todas completas) sean la realizada en prosa por Astrana Marín (1929), la de Mújica Láinez (1963) en endecasílabos blancos, la de García Calvo (1974) en tridecasílabos y la de Ehrenhaus (2009) en endecasílabos yámbicos, a la que el mismo Ehrenhaus añadió en 2018 la versión más libérrima de todas, una traducción en haikus. Dado que los Sonnets se han abordado casi de todas las formas imaginables, resultaba importante aportar un valor añadido que 
hiciese especialmente atractiva nuestra versión para que tuviese posibilidades reales de encontrar un hueco en el mundo editorial. Que el traductor tome la iniciativa constituye una práctica perfectamente normal en el mundo profesional. En este enfoque, el traductor diseña desde el principio un proyecto viable y lo propone a una editorial para su posible publicación. Dentro del amplio panorama de las retraducciones y sus posibles tendencias exotizantes, sobre el que existe un interesante debate teórico que no es posible desarrollar aquí (cf. por ej. Berman 1990, Gambier 1994, Paloposki \& Koskinen 2004, Venuti 2004, Brownlie 2006 y DeaneCox 2014), se optó por una versión tendente a la exotización que buscó sacar el máximo partido al componente académico del proyecto, proponiendo una traducción doble y comentada, de modo que el lector tuviese en sus manos varias versiones simultáneas con distinto enfoque y acompañadas de un análisis complementario de cada poema. Para dar inicio al proyecto de traducir la totalidad de los sonetos, se buscó un corpus con unidad temática que justificara su publicación independiente, por lo que se escogieron los 17 primeros poemas, un subciclo por sí mismo dentro de los sonetos de Shakespeare al girar en torno a un único leitmotiv: el deber que tienen los seres hermosos de perpetuar su belleza a través de la descendencia, lo que le ha granjeado el título de ciclo o serie de "la procreación".

Para esta oferta de doble traducción con aparato analítico, en primer lugar, se planteó su traducción en prosa como instrumento de apoyo para los muchos lectores que pudiesen estar tentados de realizar una lectura asistida a partir del original, una po- sibilidad cada vez más real en España con el 323 aumento del conocimiento del inglés que se ha producido en las últimas décadas. A ese apoyo al lector se le sumó la competencia documental que el alumnado había adquirido a lo largo de la carrera, lo que nos permitió añadir un análisis global de la retórica de cada soneto, centrándonos en las figuras más destacadas y, sobre todo, en las ambigüedades y juegos de palabras que con tanta insistencia y maestría empleaba Shakespeare. Dado que el alumnado carecía de formación filológica previa sobre Shakespeare y sus sonetos, se ofrecieron varias sesiones formativas en clase y se establecieron una serie de lecturas obligatorias compuestas por estudios de la obra original, ediciones críticas en inglés y análisis de las traducciones realizadas hasta el momento. El alumnado debía también comentar la utilidad de estas fuentes para el proyecto.

Como colofón, se decidió ofrecer también una versión poética que sintetizara la versión instrumental en prosa y los análisis previos en una versión que trataba de unificarlo todo. Dicha versión estaría centrada en el plano artístico, para que la experiencia lectora pudiese ser simultáneamente informativa (versión en prosa, sinopsis y análisis) y estética (versión rimada), quedando a criterio de cada lector la posibilidad de optar por cualquiera de los dos enfoques o, mejor aún, de elegir una experiencia acumulativa que le permitiese llegar al poema como expresión artística tras profundizar antes de manera analítica en el contenido y los recursos retóricos del soneto correspondiente.

Para la versión poética se eligió un formato, el verso alejandrino, que permite dotar a los poemas de una atmósfera clásica y que 
324

se ha cultivado con asiduidad en el soneto español desde el modernismo. Frente a los endecasílabos -el formato hispano contemporáneo de Shakespeare-, que con frecuencia exigen condensar el contenido de los pentámetros yámbicos shakespearianos, el verso alejandrino también cuenta con tradición en la traducción de los sonetos isabelinos y proporciona tres sílabas adicionales que permiten acomodar mucho mejor toda la información presente en cada verso original sin renunciar a la densidad necesaria en este tipo de composiciones. Ante la falta de espacio aquí para extenderse sobre las decisiones métricas tomadas, remitimos al lector al prólogo del volumen resultante, donde se explica con mayor detalle.

- Formación previa habilitadora por parte del profesor mediante sesiones teóricas sobre William Shakespeare y su época, así como sobre la traducción y retraducción de textos antiguos en general y de poesía clásica en particular. Se trataba de transmitir en poco tiempo (60 horas de clase) un marco conceptual complejo pero necesario para poder abordar la traducción propuesta y para poder procesar a partir de una comprensión suficiente las lecturas que el alumnado debería realizar por su cuenta. Estas clases, de carácter expositivo, se completaron con un importante número de fragmentos significativos y referencias bibliográficas sobre estos mismos asuntos, lo que en suma podríamos calificar de una recopilación de voces distintas a la del profesor a través de citas sobre las cuestiones tratadas y de propuestas bibliográficas para la profundización en los temas abordados. En total, el proceso de documentación, traducción y revisión fue de ocho meses, lo que supuso ir más allá de la duración de la asignatura, que era cuatrimestral.
- Prácticas de traducción sobre textos comparables a los del proyecto. A manera de formación previa, en concreto, se solicitó del alumnado la traducción de otros sonetos de Shakespeare que posteriormente se ponían en común en clase haciendo énfasis en la pluralidad de soluciones legítimas y el análisis detallado de la naturaleza de las diferencias que aportaba cada una, siempre con el objeto de que el alumnado adquiriese las herramientas necesarias para contemplar soluciones diversas y elegir de manera motivada entre ellas.

- Aprovechamiento de los recursos bibliotecarios y la capacidad documentalista del alumnado. El profesor realizó una búsqueda bibliográfica previa centrada en estudios de los sonetos, traducciones y estudios de las traducciones disponibles o bien a través del acceso abierto en internet o bien en las bibliotecas de nuestra universidad, ya fuera mediante suscripción electrónica o en formato impreso. Adicionalmente, el docente se puso en contacto con los responsables bibliotecarios para acordar que congelasen el préstamo de ese material durante dos meses, de manera que estuviera concentrado en un único lugar y a disposición de cualquier usuario, pero nadie pudiera sacarlo en préstamo. A continuación, se acordó con el alumnado la obligatoriedad de consultar dicho material, comprometiéndose todos los grupos a estudiar todos los materiales y utilizarlos tanto para la traducción como para el análisis de los sonetos.

Esta consulta de ediciones críticas en inglés y estudios sobre los sonetos y sus traducciones sirvió para obtener información exhaustiva sobre las particularidades de cada soneto desde una multiplicidad de voces investigadoras. Fue también muy útil para 
obtener la información específica necesaria sobre las figuras retóricas, con especial atención a los juegos de palabras, cuya traducción se había estudiado especialmente en la asignatura de traducción literaria del año anterior y que resultan tan habituales en Shakespeare. Por lo demás, la doble versión acompañada del análisis nos permitió recoger todas estas particularidades también con una vocación polifónica: explicándolas en el análisis, centrándonos en plasmar los dobles sentidos en la prosa aprovechando la inexistencia de limitaciones silábicas y tratando de reproducir la ambigüedad y riqueza interpretativa en unas versiones poéticas que podían apoyarse en todo el trabajo previo y a disposición del lector.

- Creación de grupos autónomos de alumnos con distribución rotativa de roles a modo de comunidad de prácticas, con el profesor como experto de referencia. Se distribuyó al alumnado en grupos de tres, alguno de los cuales por afinidades personales y logísticas (cercanía residencial sobre todo) se amplió a grupos de cuatro. La idea central consistía en distribuir los papeles principales de la realidad editorial profesional con el fin de profundizar en su conocimiento mediante la experiencia propia. Así, se propuso que los componentes del grupo fuesen alternativamente editora, documentalista, traductora y correctora de estilo para cada uno de los sonetos, al mismo tiempo que se daba margen de libertad para la aplicación de esta rotación, pudiendo cada grupo solicitar al profesor cambios en función de su experiencia organizativa o de sus preferencias, pero siempre interviniendo todos los miembros del grupo como cotraductoras de al menos un soneto. Este último rasgo organizativo fue el que resultó ser de menor agrado para el alumnado, que, sobre todo por razones de currículo, deseaba mayoritariamente poder firmar la traducción de los sonetos de manera individual. Este cambio de diseño se discutió en el grupo que formaba la red de innovación docente y de cara al curso siguiente, en el que se continuará con el proyecto hasta haber completado la traducción de todos los sonetos de Shakespeare, se decidió proponer que hubiese un estudiante responsable final único para cada poema, pero siempre sin abandonar la perspectiva colaborativa, basada en la revisión horizontal. En este sentido, resulta especialmente destacable esta modificación del diseño original como ilustración de que el proceso de aprendizaje no solo atañe al alumnado, sino que el proceso colaborativo también permite al docente, si mantiene los ojos y la mente abiertos, aprender mucho sobre didáctica y traducción cuando cuenta con un grupo de alumnos motivados y que se consideran dueños de su propio trabajo.

La filosofía del trabajo colaborativo en grupo radicaba en potenciar la capacidad crítica horizontal y el trabajo autónomo, al mismo tiempo que sujeto a una exigencia de calidad permanente por parte de los demás miembros del grupo en condición de pares. La idea de los roles rotativos, por su parte, buscaba que el alumnado experimentase y fuese conocedor en primera persona de las exigencias y responsabilidad de cada uno de los agentes que intervienen en una traducción literaria. Así, el editor debe ser el iniciador del proceso, proponiendo un contrato de traducción equilibrado y negociado - cuya presentación consensuada acompañada de un análisis de las condiciones laborales del traductor literario se solicitó como parte del trabajo-, además de ser 
el mediador cuando existan diferencias de criterio entre traductor y corrector. El documentalista debe ser capaz de profundizar en el texto que se va a traducir para proporcionar una interpretación intersubjetiva e informada, algo especialmente necesario en el caso de que se combinen los textos en inglés isabelino y la poesía, que se caracteriza por su densidad semántica. El corrector de estilo, por su parte, debe ser capaz de detectar problemas textuales y de presentarlos al traductor de forma proactiva, no como crítica inhabilitante, sino como ocasión de mejora, sabiendo negociar el resultado final de manera colaborativa. Tangencialmente, conviene comentar aquí que la cuestión del modo en que el alumnado efectuase y recibiese las críticas de sus compañeros era una de las que más preocupaba al docente, por la posibilidad de que se produjeran bloqueos que les impidiesen alcanzar una solución. Afortunadamente, el alumnado asimiló perfectamente el espíritu colaborativo que subyace en todo el proyecto y el procedimiento funcionó de forma muy satisfactoria: con procesos de debate y negociación reales entre el alumnado y una gran capacidad para llegar a acuerdos que siempre redundaron en un aumento de la calidad de la propuesta de traducción.

- Fijación de plazos escalonados. En primer lugar, cualquier reproducción de la realidad editorial en traducción debe otorgar una importancia central a los plazos, que constituyen una de las variables profesionales omnipresentes en la vida del traductor, hasta el punto de que es probablemente el factor laboral más comentado entre los profesionales, que siempre insisten en el carácter casi sagrado de los plazos y en la necesidad de aprender a vivir bajo el estrés que acarrean. Atendiendo al carácter didáctico de la experiencia, se establecieron tres entregas escalonadas que permitieran también al profesor supervisar y ofrecer retroalimentación para que el proceso de traducción estuviera encauzado desde un principio y no pudiera llegar a descarrilar en ningún momento. Un primer plazo requería la entrega de una traducción en prosa y el principio del análisis, además de un informe sobre la marcha del trabajo en grupo para comprobar que la documentación previa, la interpretación de los originales y el funcionamiento grupal eran correctos. Un segundo plazo requería ya la entrega del trabajo completo, con traducción poética incluida, donde jugaba un papel clave la retroalimentación del profesor en forma de señalamiento de problemas sin aportar soluciones, pero sí pistas cuando fue necesario. El tercer plazo suponía la entrega del trabajo completo revisado y una última retroalimentación del profesor, que intervino con propuestas de solución más explícitas cuando fue necesario. En este sentido, es importante hacer constar que tras esta tercera entrega de trabajo esencialmente autónomo por parte de las alumnas, los problemas residuales fueron mínimos y la calidad del producto final realmente alta.

- Presentación de la propuesta a una editorial comercial, cuya publicación suponía la mejor prueba de la adecuación del trabajo realizado, ya que implicaba poner en juego su prestigio y arriesgar una inversión económica que solo podría recuperar si las traducciones y análisis realizados eran realmente de calidad. La combinación de los dos tipos de traducción más el componente analítico, que buscaba ofrecer valor 
añadido sobre las traducciones previas, así como la supervisión académica garantizada resultaron ser factores en principio interesantes para un editor, con el que se acordó presentar el manuscrito final a partir de los parámetros aquí descritos para que, a la vista de la traducción definitiva, tomase una decisión final sobre su posible publicación. A este respecto, cabe decir con satisfacción que el trabajo colectivo produjo sus frutos y que el editor aprobó sin reservas el manuscrito. Al mismo tiempo, esta nueva etapa supuso ajustarlo a requisitos tipográficos sobrevenidos - especialmente de máximo de palabras por página- que exigieron soluciones urgentes. Creemos que resulta muy importante y revelador comentar que tanto la última entrega como el ajuste tipográfico que acabamos de mencionar se realizaron con la asignatura finalizada y las notas ya puestas. Desde un punto de vista académico, por tanto, el alumnado no se jugaba absolutamente nada en el resto del proceso. Sin embargo, esto no supuso ninguna relajación por su parte, algo que nos parece buena prueba de su alto grado de implicación en el proyecto y de la conciencia de profesionalidad que habían llegado a adquirir.

- Evaluación continua y final del proyecto. Se diseñaron seis mecanismos de evaluación del proyecto con el doble fin de realizar un seguimiento muy cercano que garantizase su viabilidad y de obtener retroalimentación que permitiese efectuar modificaciones sobre la marcha cuando fuese necesario y recopilar información para mejorar el diseño en sucesivas ediciones.

El primer mecanismo de evaluación continua y el principal desde el punto de vista didáctico consistió en el ya mencionado intento de potenciar la capacidad crítica del alumnado mediante la evaluación horizontal entre pares, que abarcaba todos los aspectos de la traducción, tanto los semánticos como los estilísticos y los analíticos. La distribución de roles en los grupos con responsabilidades individuales rotativas claramente marcadas y conducentes a establecer un reparto equitativo del trabajo estaba pensada para que el alumnado tuviese que tomar decisiones de forma autónoma bajo la supervisión de sus pares con una dinámica de debate libre entre iguales y trabajo colaborativo. Como refuerzo y sistema de control de la retroalimentación interna, también se acordó que la primera entrega se acompañaría de un pequeño informe sobre la marcha del trabajo en grupo y se insistió en que el profesor estaría disponible en cualquier momento para mediar o aconsejar en caso de necesidad.

El segundo mecanismo de evaluación continua consistió en la supervisión del profesor. Se concertaron varias citas con los grupos a continuación de cada una de las entregas, que fueron devueltas con anotaciones y sugerencias del profesor para ir mejorando los resultados desde el principio. Para que el trabajo fuese lo más autónomo posible, en todo momento, el profesor trató de no indicar soluciones finales. En este sentido, la dinámica de supervisión consistió en señalar siempre los problemas y sus posibles razones, así como vías hacia una solución que el alumnado debía explorar autónomamente. Este estadio de la revisión no formará parte habitualmente de la realidad profesional y refleja el origen académico del proyecto. Como cualquier simulación, el paralelismo con la realidad no es absoluto, pero la idea de no ofrecer soluciones sino señalar problemas va precisamente en la línea de refor- 
zar la autonomía que precisará el alumnado nada más concluir la carrera, un par de meses después de concluir este proyecto. El tercer instrumento de evaluación continua consistió en la constitución de una red de innovación didáctica compuesta por cuatro profesores de traducción literaria y dos alumnas de la asignatura. La tarea de esta comisión consistió en mantener diversas reuniones a lo largo del cuatrimestre para supervisar y enriquecer el diseño, aportando una perspectiva polifónica al desarrollo del mismo. Esta red evaluó y, tras el debate pertinente, validó el diseño inicial del proyecto y la encuesta de valoración del alumnado, supervisó su desarrollo, especialmente a través de las dos alumnas del grupo que formaban parte de la red, y valoró posibles cambios de cara a posteriores ediciones, muy especialmente la atribución individual de un soneto a cada estudiante.

La evaluación final, por su parte, se configuró a través de otros tres mecanismos: la calificación final del trabajo, una encuesta al concluir la asignatura y el juicio final externo del editor. La calificación final del trabajo, obligatoria desde el punto de vista administrativo, respondió a los cánones habituales de este tipo de juicios con el añadido de la importancia central de señalar los últimos desajustes, ya que como se ha comentado, el trabajo continuó tras concluir la asignatura, ya libres de la presión de las calificaciones.

La encuesta la diseñaron el profesor y el grupo de innovación docente para recibir retroalimentación del alumnado de cara a la asignatura y al diseño del trabajo final en años sucesivos. Cabe destacar que las respuestas eran anónimas (no había modo tampoco de saber quién había contesta- do y quién no) y que contestó el $90 \%$ del alumnado. En el apartado de Resultados se comentará brevemente la información obtenida. En el anexo que se puede encontrar al final de este trabajo se recogen todas las preguntas, con sus respuestas respectivas en términos porcentuales.

El último instrumento de evaluación, la aprobación o rechazo del editor comercial a la hora de arriesgar su dinero y, sobre todo, su prestigio en la publicación de los resultados en forma de libro impreso, resultó netamente positivo y el lanzamiento del volumen tuvo lugar a finales de junio de 2019. Al respecto, lo importante de este mecanismo es el hecho de que sea externo y, por tanto, especialmente objetivo. El editor no tiene ningún interés personal en el libro y su decisión final se tomó tras recibir el manuscrito, en el que ha visto calidad y posibilidades comerciales. Tanto es así que ya está apalabrada la continuidad del proyecto durante tres años más, hasta traducir la totalidad de los 154 sonetos que publicó William Shakespeare.

\section{RESULTADOS}

El proyecto de traducción de los sonetos de Shakespeare ha constituido una experiencia provechosa de la que cabe destacar los resultados siguientes:

- La incorporación de un enfoque con un fuerte componente horizontal y cooperativo basado en la comunidad de prácticas es viable, especialmente con alumnado de último curso del Grado, que están ya dotados de las competencias necesarias para abordar un proyecto profesional en sentido pleno. Traducir la poesía de Shakespeare aplicando mecanismos métricos clásicos constituía un reto importante del que to- 
dos los traductores literarios profesionales no saldrían airosos, por lo que este aspecto constituía uno de los principales temores del docente. Sin embargo, una de las respuestas de la encuesta indica que un $78 \%$ del alumnado considera el trabajo de una dificultad adecuada. En esta misma línea, el $22 \%$ de respuestas que sí indican que puede ser más complejo de la cuenta refuerzan la idea de que se trata de un proyecto exigente, pero viable, especialmente con un enfoque colaborativo, donde el alumnado con más dificultades no se sienta en ningún momento perdido. Estaríamos en ese sentido en lo que a partir de Vygotsky se denomina zona de desarrollo próximo, que sería la más adecuada para el progreso en el aprendizaje.

- De la encuesta realizada, resulta especialmente interesante destacar tres resultados más: en primer lugar, que casi un $90 \%$ de las alumnas cree que el trabajo es mucho más provechoso en grupo (no hubiesen preferido hacerlo de manera individual); en segundo lugar, que la conexión con el mundo profesional en forma de producto publicable constituye un elemento central en la motivación (94\%); en tercer y último lugar, las respuestas indican su sensación de que el aprendizaje con la asignatura les había parecido claramente superior a lo habitual con una docencia más tradicional.

- En el capítulo de las mejoras para años venideros, debe indicarse a partir de las conversaciones con el alumnado que se ha detectado la conveniencia de aumentar la responsabilidad individual en el seno de cada grupo, asignando al menos un soneto completo a cada alumno como traductor individual - siempre dentro del entramado horizontal del grupo como sistema de maduración y control de calidad-.
- El último y gratificante resultado del proyecto es el libro mismo, que ya ha salido a la luz en la editorial zaragozana Libros del Innombrable bajo el título de Sonetos para tener un hijo. Sonetos I, un nombre que constituye una promesa de continuidad. Aparte de la experiencia didáctica, este libro constituirá una tarjeta de presentación de cada alumna, que podrá salir al mercado laboral con experiencia profesional real en su currículo. Desde un punto de vista psicológico, creemos que haber salido airosas de este desafío profesional puede suponer también un plus de confianza que esperamos que resulte beneficioso en su posterior desempeño profesional.

\section{CONCLUSIÓN}

Como se ha tratado de mostrar en el apartado anterior y reflejan los distintos instrumentos de seguimiento y evaluación aplicados, este proyecto colaborativo de traducción poética ha cumplido sus expectativas, en gran medida gracias a un diseño centrado en el alumno que ha logrado suscitar su entusiasmo y la implicación activa de todos los agentes del proceso educativo.

En gran parte hemos basado nuestro proyecto en el aprendizaje colaborativo, también conocido como Project-Based Learning (PBL) que, según afirma Lobato (2013), fomenta la autonomía y el desarrollo de la capacidad crítica del alumnado. Como la misma autora explica, con este enfoque los alumnos pasan a situarse en el centro de su aprendizaje, convirtiéndose en los principales protagonistas del mismo. Así, nuestras alumnas han pasado a ser agentes activos responsables de organizarse, documentarse y encargarse de resolver las dificultades encontradas en el camino. Del mismo modo, han sabido reconducir aquellos problemas y aspectos susceptibles de 
330 mejora señalados por el profesor de la asignatura. De esta forma, se ha fomentado el trabajo en grupo, asumiendo los diferentes roles que forman parte de la realidad profesional del traductor.

Además, merece la pena incidir en el hecho de haber usado materiales auténticos, lo que ha servido como motivación muy clara al $94 \%$ de nuestro alumnado. Kelly (2005) insiste en la utilidad de la simulación de proyectos reales en el aula de Traducción, pero piensa que son recomendables en los cursos superiores. En nuestro caso, como hemos comentado, el proyecto se ha desarrollado en el último curso del Grado y no se ha limitado a una simulación. Antes hacíamos mención del enfoque constructivista que defiende Kiraly (2001) a través de la participación activa del alumnado, un objetivo que, sin duda, se ha conseguido en esta propuesta. Nuestras alumnas, como comentaba el propio Kiraly (2005), se han sentido empoderadas ante la realización de un proyecto real colaborativo en el que se han enfrentado a situaciones y problemáticas similares a las que vivirán en una carrera profesional a cuyas puertas muchas de ellas se encuentran ya.

Quizá el aspecto que más convendría comentar llegados a este punto es una de las cuestiones que más suele preocupar al profesorado cuando se le habla de delegar parte de las decisiones y la evaluación de resultados en el alumnado. Por ello, es necesario destacar aquí la importancia de la labor docente, que continúa siendo imprescindible y central en una perspectiva didáctica colaborativa y de vocación horizontal. En este sentido, parece obvio que indicarle al alumnado que actúe por su cuenta sin preparación previa, supervisión ni un marco de actuación claro sería una receta segura hacia el fracaso. Así, además de ser el guía del proceso de enseñanza-apren- dizaje, el profesor facilitador continúa actuando como experto imprescindible, de cuya decisión inicial y supervisión proactiva depende el éxito de cada proyecto didáctico, todo ello sin renunciar a estrategias más tradicionales de enseñanza, especialmente en las primeras fases del proyecto, donde la escasez de tiempo disponible y el notable volumen de conocimiento y destrezas que se deben adquirir aconsejan una transmisión directa profesor-alumnado. No debemos olvidar que el alumnado, sobre todo al principio de cada asignatura, carece de parte de las herramientas y conocimientos cuya adquisición constituye precisamente el objeto principal de su presencia en clase. Corresponde pues al profesor diseñar iniciativas sólidas y minuciosamente planeadas que permitan a ese mismo alumnado levantar progresivamente el vuelo para conseguir la autonomía que finalmente le permitirá ejercer su labor de manera experta y con una base científica con la que armarse para ser consciente de sí mismo como profesional, como corresponde a la formación universitaria.

\section{REFERENCIAS}

Berman, Antoine (1990): «La retraduction comme espace de la traduction», Palimpsestes, 4, 1-7.

Brownlie, Siobhan (2006): «Narrative Theory and Retranslation Theory», Across Languages and Cultures, 7:2, 145-170.

Deane-Cox, Sharon (2014): Retranslation. Translation, Literature and Reinterpretation, London: Bloomsbury.

Durán, David (2014): Aprenseñar. Evidencias e implicaciones educativas de aprender enseñando, Madrid: Síntesis.

GAMbier, Yves (1994): «La retraduction, retour et détour», Meta, 39:3, 413-417.

GonzÁLez Davies, María \& Vanessa Enríquez Raído (2016): Situated learning in translator and interpreter training: bridging research and good practice. The Interpreter and Translator Trainer (ITT) 10/1. 
Johnson, David W. \& Roger T. Johnson (1990): «Cooperative learning and achievement», en Shlomo Sharan (ed.), Cooperative learning: Theory and research, 23-37, New York: Praeger Publishers.

KelLy, Dorothy (2005): A Handbook for Translator Trainers, Mánchester: St Jerome.

KIraly, Donald Charles (1995): Pathways to Translation. Pedagogy and Process, Kent: Kent State University Press.

KiraLY, Donald Charles (2001): «Towards a Constructivist Approach to Translator Education», Quaderns. Revista De Traducció, 6, 50-53.

KIraly, Donald Charles (2005): «Project-Based Learning: A Case for Situated Translation», Meta, 50/4, 1098-1111.

KIRALY, Donald Charles (2014): «From Assumptions about Knowing and Learning to Praxis in Translator Education», inTRAlinea, (s.p.).

LAVE, Jean \& Etienne Wenger (1991): Situated Learning: Legitimate Peripheral Participation, Cambridge: Cambridge University Press.

Lobato Patricio, Julia (2013): «Propuesta didáctica para las clases de traducción especializada: El aprendizaje basado en proyectos», Tonos Digital, 25, (s.p.).

Marco Borillo, Josep (2016): «On the margins of the profession: the work placement as a site for the literary translator trainee's legitimate peripheral participation», The Interpreter and Translator Trainer (ITT), 10/1, 29-43.

Paloposki, Outi \& Kaisa Koskinen (2004): «A Thousand and One Translations: Revisiting Retranslation», en Gyde Hansen, Kirsten Malmkjaer \& Daniel Gile (eds.), Claims, Changes and Challenges in Translation Studies, 27-38 Ámsterdam: John Benjamins.

PIeTrZAK, Paulina (2014): «Towards Effective Feedback to Translation Students: Empowering Through Group Revision and Evaluation», inTRAlinea (Special Issue) (s.p.).

Venuti, Lawrence (2004): «Retranslations. The Creation of Value», Bucknell Review, 47:1, 25-38.

\section{ANEXOS}

Se recoge aquí la encuesta final, incluyendo los porcentajes correspondientes a cada respuesta, realizada al alumnado tras concluir la asignatura. Respondió, siempre de forma anónima, el 90\% (20 de 22) del grupo.

I. La asignatura Traducción Literaria Avanzada Inglés (32651) es optativa. ¿Cuál es la razón principal de que te hayas matriculado?
a. Completar el itinerario de Traducción especializada $-5,6 \%$
b. Interés por la traducción literaria $-77,8 \%$
c. Por ninguna razón en especial - 16,6\%

2. ¿Cuántas obras literarias lees de media al año?
a. $1-3-44,4 \%$
b. $4-6-27,8 \%$
c. $7-10-16,7 \%$
d. Al menos una al mes - 11,1\%

3. Marca por favor aquellas áreas de las que antes de iniciar la asignatura tuvieras conocimientos previos para al menos mantener una conversación formal sobre ellas.
a. Teoría e historia de la literatura - 83,3\%
b. Teoría de la traducción literaria - 50,0\%
c. Crítica de traducción - 33,3\%
d. Versificación - 16,7\%
e. Mundo editorial - 22,2\%
f. Profesión de traductor literario - 27,8\%

4. Después de cursar la asignatura, ¿tienes conocimientos sobre alguno de estos ámbitos que ahora sí te permitan mantener una 
conversación formal? Marca únicamente los que sean nuevos en este sentido.

a. Teoría e historia de la literatura $-61,1 \%$

b. Teoría de la traducción literaria $-72,2 \%$

c. Crítica de traducción $-33,3 \%$

d. Versificación $-61,1 \%$

e. Mundo editorial - 50,0\%

f. Profesión de traductor literario - 55,6\%

5. ¿Ha cambiado tu opinión sobre la traducción literaria después de cursar la asignatura?

a. Me gustaba antes y continúa gustándome ahora. $-38,9 \%$

b. Me gustaba antes y ahora me gusta más aún. $-33,3 \%$

c. Antes no me llamaba mucho la atención y ahora me gusta. $-27,8 \%$

d. Antes me gustaba, pero ahora no me atrae tanto. $-0 \%$

6. El proyecto de traducción editada de los Sonetos de Shakespeare, te parece:

a. Un trabajo final de asignatura más. $-0 \%$

b. Un trabajo demasiado exigente para ser el final de una asignatura. - $0 \%$

c. Un trabajo especialmente motivador en sí mismo. $-5,6 \%$

d. Un trabajo especialmente motivador en sí mismo, pero especialmente por la posibilidad de verlo publicado en un libro profesional. - $94,4 \%$

7. El proyecto de traducción editada de los Sonetos de Shakespeare debería ser un trabajo:
a. Individual $-11,1 \%$
b. Colectivo $-88,9 \%$

8. El texto elegido para la traducción editada:

a. Es adecuado en cuanto a dificultad y por la gran repercusión del autor elegido y la bibliografía de apoyo existente. $-77,8 \%$

b. Es demasiado difícil y debería elegirse un autor más sencillo. $-22,2 \%$

9. En cuanto al texto elegido para la traducción editada:

a. Los poemas breves, tipo soneto, son los más adecuados por poderse compartimentar fácilmente. $-55,6 \%$

b. Sería más adecuado elegir relatos breves o incluso una novela, porque resultan más sencillos y modernos, aunque hubiese que compartimentar cada texto entre varios alumnos. $-44,4 \%$

I0. ¿Crees que la asignatura de Traducción Literaria Avanzada debería dedicarse únicamente a la traducción poética?

a. Sí, la traducción de narrativa ya se ve suficientemente en la asignatura obligatoria de traducción literaria de tercero y necesitamos el máximo tiempo de prácticas en la modalidad poética. $-5,6 \%$

b. No, la traducción de narrativa debe continuar practicándose en la asignatura de avanzada por su gran importancia. $-94,4 \%$

II. La elaboración y análisis de un contrato de traducción me ha servido:

a. De poco o nada, porque ya conocía la dinámica de este tipo de acuerdos profesionales. $-5,6 \%$ 
b. Mucho, para situarme con mayor conocimiento de causa de cara al mercado profesional de la traducción. $-94,4 \%$. 